\title{
Incidences of fatal postoperative pulmonary embolism after prophylaxis with dextran 70 and low-dose heparin: an international multicentre study
}

\author{
U F GRUBER, T SALDEEN, T BROKOP, B EKLÖF, I ERIKSSON, I GOLDIE, L GRAN, \\ M HOHL, T JONSSON, S KRISTERSSON, K G LJUNGSTRÖM, T LUND, H MAARTMAN MOE, \\ E SVENSJÖ, D THOMSON, J TORHORST, A TRIPPESTAD, M ULSTEIN
}

\section{Summary and conclusions}

A total of 4352 patients were admitted to a prospective' randomised multicentre trial comparing the prophylactic efficacy of dextran 70 and low-dose heparin against fatal pulmonary embolism after elective operations for general, orthopaedic, urological, and gynaecological conditions. Out of 3984 patients correctly admitted, 1993 were allocated to receive dextran 70 and 1991 to receive low-dose heparin.

Withdrawal of prophylaxis because of bleeding or technical difficulties occurred more often in the heparin group, but allergic reactions were more common in the dextran group. Of the 75 patients who died within 30 days after operation, 38 had been given dextran and 37 lowdose heparin. Necropsy was performed in 33 and 32 of these cases respectively. In six patients in each group pulmonary embolism was the sole or a contributory cause of death. Of these, five patients in the dextran group and two in the heparin group had received a full course of prophylaxis.

There was no statistically significant difference between the two treatment groups in the incidence of fatal pulmonary embolism after a full course of prophylaxis.

\section{Introduction}

Dextran and low-dose heparin are claimed to reduce the incidence of postoperative thromboembolic complications. ${ }^{1-4}$ In 1975 a leading article in the $B M \mathcal{F}^{5}$ stated: "A large scale multicentre trial to compare in surgical patients the effects of sodium heparin and dextran in the prevention of fatal pulmonary embolism is now needed."

We have investigated the incidence of fatal pulmonary embolism within 30 days after general, gynaecological, urological, and orthopaedic surgery in patients given either dextran or heparin prophylaxis. Advantages and disadvantages of each regimen and the incidence of side effects were also evaluated. Eight hospitals participated: five in Sweden, two in Switzerland, and one in Norway.

\section{Patients and methods}

Patients aged over 40 who were scheduled for elective operations likely to last more than 30 minutes under a general anaesthetic were eligible for inclusion in the study. They were divided into four surgical subgroups: general, gynaecological, urological, and orthopaedic. If any patient required a second operation within one month and prophylaxis was not contraindicated the same prophylactic regimen was used but the follow-up time was taken from the first operation. For ethical reasons an untreated control group was not included.

Patients were allocated at random to each prophylactic group by using numbered sealed envelopes. Original trial forms were kept at each centre and copies forwarded to the co-ordinating centre (Institute of Forensic Medicine, University of Uppsala). The information was recorded on punch cards and analysed with a Hewlett-Packard 2100 computer. A record of the prearranged sequence of numbered envelopes was kept at the co-ordinating centre. When the study was completed allocation of patients to each group was checked against this record to confirm that randomisation had been successful. The study was carried out during June 1976 to June 1978 .

Dextran-Three $500 \mathrm{ml}$ infusions of dextran $70,6 \%$ in saline (Macrodex), were given. The first was begun soon after induction of anaesthesia and completed within about an hour. The second was started after the operation and completed within about two hours. The third was given on the first postoperative day over two to four hours.

Heparin-Subcutaneous sodium heparin 5000 IU was given two hours preoperatively and eight-hourly thereafter for six postoperative days or for the duration of hospital stay if under seven days. Three preparations were used: Liquemin in Switzerland, Heparin Novo in Norway, and Heparin Vitrum in Sweden. If the patient was confined to bed for over seven days heparin was continued until the patient was fully mobilised. A 26-gauge needle was used and the heparin injected into the anterior abdominal wall, as described. ${ }^{6}$

Assessment-The incidence of pulmonary embolism found at 
necropsy was used as the sole criterion of failure of prophylaxis. The pulmonary arteries were dissected down to small segmental vessels. Immediately after each necropsy a report was sent to the co-ordinating centre for prompt analysis. Cases of pulmonary embolism were divided into three groups: (a) those in which no other cause of death was found, $(b)$ those in which pulmonary embolism was considered to be a contributory cause of death, and $(c)$ those in which pulmonary embolism was regarded as incidental and not having contributed to death-for example, old or single smaller emboli. If deep vein thrombosis or pulmonary embolism was diagnosed clinically the patient was treated in the usual way at the particular centre. Intraoperative blood loss was estimated by the surgeon or anaesthetist or both. Surgical wounds and heparin injection sites were examined for haematomas. Also the preoperative haemoglobin concentration and lowest value recorded during the first postoperative week were noted together with the amount of blood transfused. Possible allergic reactions to dextran were classified as described. ${ }^{7}$

Follow-up-All patients were followed up one month after operation whether they were still in hospital or discharged and questioned about their current condition.

Statistical methods-The $\chi^{2}$ test and Fisher's exact test were used as described. ${ }^{8}$

\section{Results}

Envelopes were opened for 4352 patients (see table Im (miniprint)), of whom 1245 were seen in Bergen, 1239 in Basle (two hospitals), 582 in Danderyd, 534 in Lund, 372 in Uppsala, 328 in Helsingborg, and 52 in Gothenburg. Of these, 2159 were allocated to receive dextran and 2193 to receive heparin. A total of 368 patients-166 in the dextran group and 202 in the heparin group-however, were wrongly admitted to the study. Of the remaining 3984 patients, 1993 were allocated to the dextran group and 1991 to the heparin group (table Im); $2627(66 \%)$ underwent general surgery, $760(19 \%$ gynaecological surgery, $414(10 \%)$ urological surgery, and $183(5 \%)$ orthopaedic surgery.

Institute of Forensic Medicine, University of Uppsala, S-752 37 Uppsala, Sweden

T SALDEEN, MD, professor of forensic medicine; co-ordinator of trial

Haukeland Sykehus, University of Bergen, N-5016 Bergen, Norway

L GRAN, MD, associate professor of anaesthesiology

$T$ LUND, MD, senior registrar in anaesthesiology

H MAARTMAN MOE, MD, senior registrar in pathology

A TRIPPESTAD, MD, associate professor of surgery

$M$ ULSTEIN, MD, associate professor of obstetrics and gynaecology

Kantonspital, University of Basle, CH-4031 Basle, Switzerland

U F GRUBER, PD, DR MED, Leitender Arzt Chirurgie

J TORHORST, PD, DR MED, pathologist

Surgical Clinic, Danderyd Hospital, S-182 03 Danderyd, Sweden

K G LJUNGSTRÖM, MD, senior registrar

Lasarettet, University of Lund, S-221 85 Lund 5, Sweden

$T$ BROKOP, MD, surgeon

B EKLÖF, MD, associate professor of surgery

D THOMSON, MD, associate professor of anaesthesiology

Frauenspital, University of Basle, $\mathrm{CH}-4031$ Basle, $S$ iizerland M HOHL, DR MED, oberarzt

Akademiska sjukhuset, University of Uppsala, S-750 14 Uppsala, Sweden

I ERIKSSON, $M D$, associate professor of surgery

Lasarettet Helsingborg, S-251 87 Helsingborg, Sweden

$S$ KRISTERSSON, MD, associate professor of surgery

Sahlgrenska sjukhuset, Department of Orthopaedic Surgery, University of Gothenburg, S-413 45 Gothenburg, Sweden

I GOLDIE, MD, associate professor of orthopaedic surgery

T JONSSON, MD, orthopaedic surgeon

Department of Experimental Medicine, Pharmacia AB, Box 604, 75125 Uppsala, Sweden

E SVENSJÖ, PHD, associate professor of pharmacology
COMPARISON OF GROUPS

The two groups were well matched for mean age (59 years), sex distribution, mean weight $(68 \mathrm{~kg})$, mean duration of operation (two hours), mean duration of anaesthesia (two hours 45 minutes), and mean duration of hospital stay ( 15 days). There was no difference in the types of operations performed between the two groups; 1132 patients in the dextran group $(57 \%$ ) and 1137 in the heparin group $(57 \%)$ underwent laparotomy.

\section{OVERALL MORTALITY RATE}

Seventy-five patients $(1.9 \%)$ died within 30 days after operation (table IIm). Of these, 38 were in the dextran group and 37 in the heparin group, and 33 and 32 respectively came to necropsy. In some cases several contributory causes of death were identified (table IIm).

\section{FINDINGS AT NECROPSY}

Five patients in the dextran group and three in the heparin group had pulmonary embolism as the sole cause of death (table III). One patient in the dextran group and three in the heparin group had pulmonary embolism as a contributory cause of death. In two dextran-treated and three heparin-treated patients incidental pulmonary embolism was found. No death occurred from pulmonary microembolism. There was no difference between the two groups in the location of emboli within the lungs.

TABLE III-Details of patients dying from pulmonary embolism

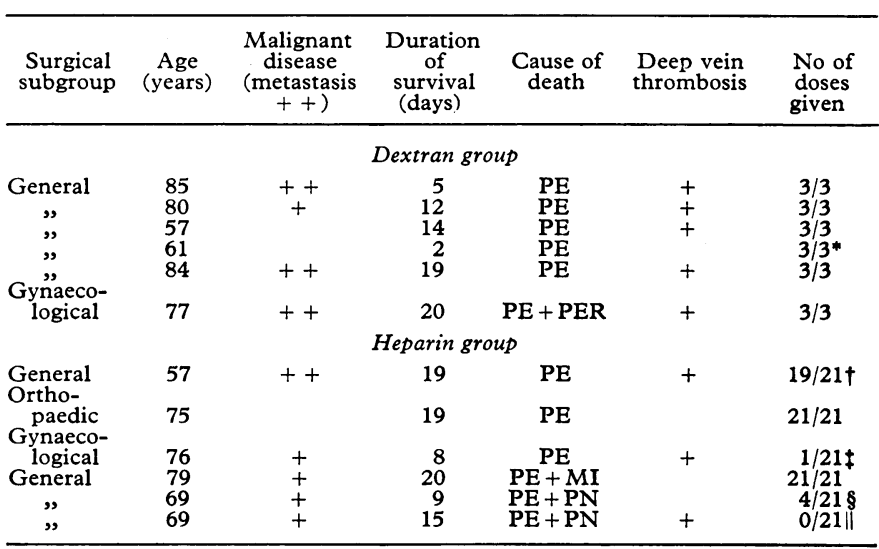

$\mathrm{PE}=$ Pulmonary embolism. $\mathrm{PER}=$ Peritonitis. $\mathrm{MI}=$ Myocardial infarction. $\mathrm{PN}=$ Pneumonia.

-Dextran was begun one and a half hours after induction of anaesthesia and 15 minutes after start of operation.

†First two doses missed.

Prophylaxis stopped owing to risk of haemorrhage.

§rophylaxis stopped because of haemorrhage.

No prophylaxis given owing to error.

Four of the dextran-treated patients had malignant disease, which in three cases had metastasised. Five patients in the heparin group had malignant disease, but only one showed evidence of metastasis.

Deep vein thrombosis was present in six patients in the dextran group and five in the heparin group. In three of the dextran-treated patients thrombosis occurred only in calf veins, and in two it was also found in femoral veins; the other patient had pelvic-vein thrombosis. In the five heparin-treated patients thrombosis was confined to the femoral veins. Of all 11 patients with thrombosis, 10 also had pulmonary emboli. In the remaining patient, who was given heparin, thrombosis occurred without pulmonary embolism.

\section{ADHERENCE TO PROTOCOL}

Of the 3984 patients who were correctly admitted to the study, 449 were not treated according to the protocol (table IVm). Reasons included technical faults and objections from patients or surgeons, both of which were more common in the heparin group than in the dextran group $\left(\chi^{2}=22.27, P<0.001 ; \chi^{2}=13.39, P<0.001\right)$. In six dextran-treated and 94 heparin-treated patients prophylaxis was 
discontinued because of haemorrhage or risk of haemorrhage ( $\left.\chi^{2}-80.56 ; P<0.001\right)$, mean blood losses during operation being $608 \mathrm{ml}(150-1600 \mathrm{ml})$ and $902 \mathrm{ml}(0-6000 \mathrm{ml})$ in the six and 94 patients respectively. In a further six dextran-treated and 22 heparintreated patients prophylaxis was interrupted by reoperation for haemorrhage $\left(\chi^{2}=8.39 ; P<0.01\right)$. These patients are included in those who underwent reoperation listed in table $\mathrm{Vm}$.

In 22 patients given dextran the infusion was discontinued because of allergic reactions probably caused by the drug. Twelve patients had grade I reactions (mild), five grade II, four grade III, and one grade IV. In the last patient the operation was cancelled. All patients recovered uneventfully from these reactions. In the heparin group three patients exhibited allergic skin reactions resulting in withdrawal of the drug.

\section{COMPLETENESS OF PROPHYLAXIS}

Complete prophylaxis was defined as the infusion of all three doses of dextran or the injection of all but two doses of heparin during the hospital stay. If the first two doses of heparin were missed, however, prophylaxis was regarded as incomplete.

Of the 1875 patients in the dextran group for whom the protocol was followed correctly, $1760(94 \%)$ received complete prophylaxis (table IVm) and $85(5 \%)$ were given only two infusions; for the remainder the information was missing. Of the 1660 heparin-treated patients, $1418(85 \%)$ were given complete prophylaxis, but only $1179(71 \%)$ received all the injections prescribed. A total of 1563 patients in the heparin group $(94 \%)$ were given over two-thirds of the doses scheduled.

Of the 1760 patients in the dextran group given complete prophylaxis, 22 received further infusions, most of which were given during one to two days. Of the 1418 patients given a complete course of heparin, 76 received additional injections, most of which were also given over one or two days.

When the incidence of fatal pulmonary embolism in patients given complete prophylaxis was compared, five of the 1760 dextran-treated patients and two of the 1418 heparin-treated patients had such emboli. This difference was not significant (Fisher's exact test, $\mathrm{P}=0.32$ ).

\section{TREATMENT OF DEEP VEIN THROMBOSIS}

Deep vein thrombosis was diagnosed clinically in 26 patients correctly admitted to the study. Of these, 18 were in the dextran group and eight in the heparin group. Only three of the dextrantreated patients had the diagnosis confirmed by phlebography or

\section{MINIPRINT TABLES}
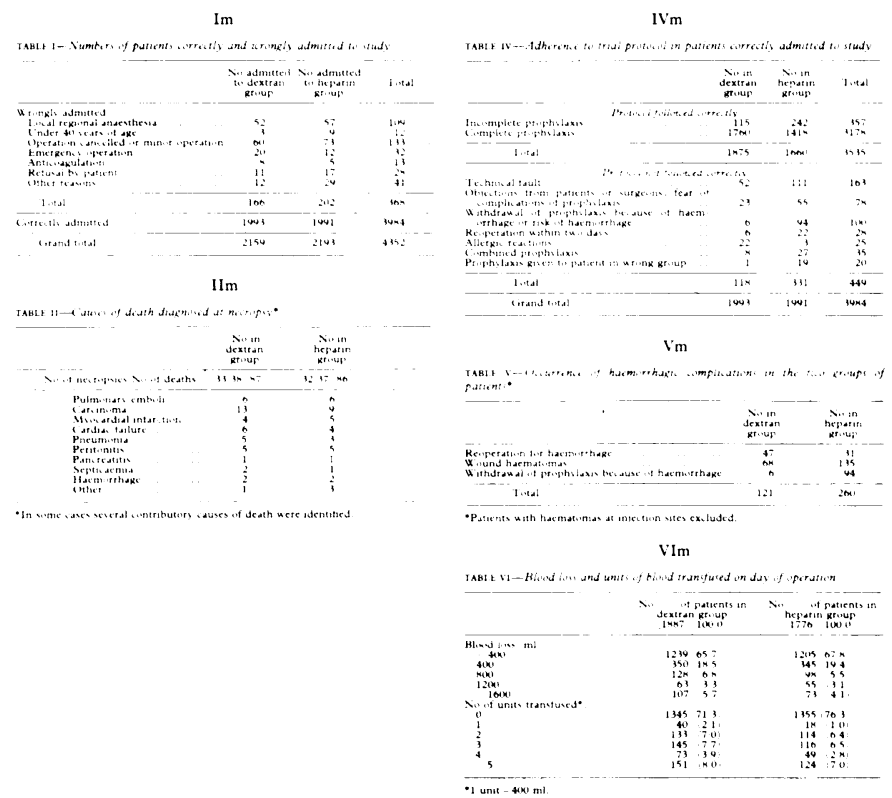

fibrinogen uptake test. Nevertheless, nine of the dextran-treated patients and six of the heparin-treated patients were given heparin or oral anticoagulants. This difference was not significant.

\section{REOPERATION}

Eighty-four patients in the dextran group and 56 in the heparin group were reoperated on for various reasons within 30 days of the initial operation. In 47 and 31 of these patients respectively the second operation was for haemorrhage (table Vm). In the remaining 37 and 25 patients in the two groups reoperation was not related to haemostasis $\left(\chi^{2}=2 \cdot 35 ; \mathrm{P}=0 \cdot 12\right)$.

\section{HAEMORRHAGIC COMPLICATIONS}

The 78 reoperations performed because of haemorrhage (47 in the dextran group, 31 in the heparin group; table $V m$ ) included ligation of bleeding vessels, evacuation of wound haematomas, and procedures for diffuse postoperative bleeding $\left(\chi^{2}=3.33 ; \mathrm{P}=0.07\right)$. These patients included those shown in table IVm in whom prophylaxis was interrupted by reoperation. Seventy-two of the reoperations $(92 \%)$ were carried out within two days. Significantly more wound haematomas occurred in the heparin group $\left(\chi^{2}=23.30 ; \mathrm{P}<0.001\right)$, and this group fared significantly worse when the incidences of all haemorrhagic complications were considered (table Vm; $\chi^{2}=56.91 ; \mathrm{P}<0.001$ ). Furthermore, 153 heparin-treated patients had haematomas at the site of injection.

\section{BLOOD LOSS AND BLOOD TRANSFUSION REQUIREMENTS}

Blood loss during operation was similar in the two treatment groups $\left(\chi^{2}=7 \cdot 13, P>0.1\right.$; table VIm). Nevertheless, 502 patients in the dextran group $(27 \%)$ as compared with 403 in the heparin group $(23 \%)$ were given two or more units of blood on the day of operation $\left(\chi^{2}=16.90 ; P<0.01\right)$. These patients include the six in the dextran group and 94 in the heparin group in whom prophylaxis was stopped because of haemorrhage, and the six in the dextran group and 22 in the heparin group in whom prophylaxis was interrupted by reoperation. Blood transfusion requirements after the day of operation were the same in the two groups. In the dextran group the haemoglobin concentration fell from a preoperative value of 13.0 SD $1.0 \mathrm{~g} / \mathrm{dl}$ to $11.0 \pm 1.0 \mathrm{~g} / \mathrm{dl}(\mathrm{n}=1359)$; the same values were observed in the heparin group $(\mathrm{n}=1250)$.

\section{ONE-MONTH FOLLOW-UP}

One month after operation there was no difference in the incidence of chest pain, respiratory difficulty, haemorrhage, haemoptysis, or wound infections between the two groups.

\section{Discussion}

Low-dose heparin and dextran reduced the incidence of fatal pulmonary embolism in two multicentre trials, ${ }^{12}$ thus confirming the results of many smaller studies. ${ }^{36}$ In our series pulmonary embolism was the sole cause of death in three patients allocated to receive heparin prophylaxis. This incidence $(0 \cdot 15 \%)$ agrees with the findings of Kakkar et $a l^{2}(0 \cdot 10 \%)$ and Kiil et $a l^{9}(0 \cdot 15 \%)$. The total mortality rate in our study was only half that reported by Kakkar et al despite the fact that we followed up all patients for one month. Follow-up time was not defined in their study, but it appears to have been shorter.

We found that the two methods of prevention-namely, three units of dextran 70 over two days and thrice-daily injections of heparin for one week-resulted in the same overall incidence of fatal pulmonary embolism. Of all 12 such cases, two of the six patients in the heparin group and five of the six in the dextran group received prophylaxis according to the protocol. Although this difference was not significant, it might suggest that a full course of heparin treatment would result in a lower incidence of fatal pulmonary embolism than the dextran regimen. 
Dextran prophylaxis continued beyond the first two days in immobilised patients may also result in a lower incidence. ${ }^{1011}$ Dextran reduces inhibition of fibrinolysis, ${ }^{12}$ which is maximal three to six days postoperatively ${ }^{13}$; hence further doses might be of value.

Allergic reactions occurred in 22 patients $(1 \cdot 1 \%)$ in the dextran group. This was a higher incidence than that reported by Ring and Messmer ${ }^{14}(0.07 \%)$, but their findings were based on units infused and not on numbers of patients treated. Since preformed dextran-reactive antibodies are reportedly of pathogenic importance in dextran reactions, ${ }^{7}$ application of the hapten-inhibition principle may offer a method of protection. A multicentre trial in seven of the eight hospitals in this study is in progress to evaluate the effect of hapten prophylaxis on allergic reactions. Preliminary findings from over 15000 patients suggest that severe dextran-induced reactions can be prevented.

Although the number of reoperations for haemorrhage was higher in the dextran group, this difference was not significant. When the incidences of all haemorrhagic complications were considered (table Vm) patients in the heparin group fared significantly worse. An explanation may be that prophylaxis with heparin was more readily withdrawn from patients prone to haemorrhage. The two treatment groups lost similar amounts of blood during operation, yet patients in the dextran group were given a larger number of transfusions. Dextran is a plasma expander, however, and decisions to transfuse may have been based on decreased packed cell volume.

The greater number of failures to give a full course of prophylaxis in the heparin group was probably partly accounted for by the number of injections required (21). Dextran infusions form part of normal volume replacement during and after operation and entail no extra work. Possibly heparin could be given more conveniently by, say, jet injection or inhalation.

We conclude that prophylaxis with dextran and low-dose heparin results in the same overall incidence of fatal pulmonary embolism.

We thank the surgeons, pathologists, and nursing staff of the participating hospitals for their co-operation; Barbro Eriksson for designing the computer program; and Annie Lindbom and Eeva Salo for excellent secretarial work. This investigation was supported by grants from the Swedish Medical Research Council (Tom Saldeen) and Fortia AB, Uppsala, Sweden.

Correspondence should be addressed to: Professor Tom Saldeen, Institute of Forensic Medicine, University of Uppsala, Dag Hammarskjölds väg 19, S-752 37 Uppsala, Sweden.

\section{References}

1 Kline A, Hughes LE, Campbell H, Williams A, Zlosnick J, Leach KG. Dextran 70 in prophylaxis of thromboembolic disease after surgery: a clinically oriented randomised double-blind trial. $\mathrm{Br} M e d \mathcal{F} 1975$;ii: 109-12.

${ }^{2}$ Kakkar VV, Corrigan TP, Fossard DP. Prevention of fatal postoperative pulmonary embolism by low doses of heparin: an international multicentre trial. Lancet 1975 ;ii:45-51.

3 Gruber UF. Dextran and the prevention of postoperative thromboembolic complications. Surg Clin North A $1975 ; 55$, No 3:679-96.

4 Bergentz S-E. Dextran in the prophylaxis of pulmonary embolism. World f Surg 1978;2:19-25.

$5 \mathrm{Br}$ Med $\mathcal{F}$ 1975;iii :447-8.

6 Kakkar V. The current status of low-dose heparin in the prophylaxis of thrombophlebitis and pulmonary embolism. World f Surg 1978;2:3-18.

7 Hedin H, Richter W, Ring J. Dextran-induced anaphylactoid reactions in man. Role of dextran-reactive antibodies. Int Arch Allergy Appl Immunol 1976;52:145-59.

${ }^{8}$ Snedecor GW, Cochrane WG. Statistical methods. Iowa: Iowa State University Press AMES, 1967.

${ }^{9}$ Kiil J, Axelsen F, Kiil J, Andersen D. Prophylaxis against postoperative pulmonary embolism and deep-vein thrombosis by low-dose heparin. Lancet 1978;i:1115-6.

10 Johnson SR, Bygdeman S, Eliasson R. Effect of dextran on postoperative thrombosis. Acta Chir Scand 1968; suppl 387:80-2.

11 Evarts CM, Feil EI. The influence of low-molecular-weight dextran on venous thrombosis following elective hip surgery. $\mathcal{F} A M A$ 1970;212:872.

12 Carlin G, Saldeen T. Effect of dextran on fibrinolysis inhibition activity in serum. Thromb Res 1978;12:1165-75.

13 Bagge L, Saldeen T. The primary fibrinolysis inhibitor and trauma. Thromb Res 1978;13:1131-6.

14 Ring J, Messmer K. Incidence and severity of anaphylactoid reactions to colloid volume substitutes. Lancet 1977;i:466-9.

(Accepted 3 October 1979)

\title{
Trimethoprim resistance in Finland after five years' use of plain trimethoprim
}

\author{
P HUOVINEN, P TOIVANEN
}

\section{Summary and conclusions}

A total of 1388 urinary bacterial pathogens were tested for resistance to plain trimethoprim after five years' use of this drug for prophylaxis against urinary tract infections. Samples were obtained in Turku, Finland, where use of the drug is much greater than in other parts of Finland.

Resistance to trimethoprim ( $\geqslant 8 \mathrm{mg} / \mathrm{l}$; agar-dilution method) occurred in $20 \cdot 3 \%$ of strains isolated from outpatients and $39.8 \%$ of strains isolated from inpatients. Escherichia coli and Micrococcus showed low incidences of resistance $(11 \%$ and $13 \%$ respectively in outpatients and $23 \%$ and $19 \%$ respectively in inpatients); Enterobacter, Streptococcus faecalis, and Staphylococcus

\footnotetext{
Department of Medical Microbiology, Turku University, SF-20520 Turku 52, Finland

P HUOVINEN, CAND MED, research associate

$\mathrm{P}$ TOIVANEN, MD, professor of bacteriology and serology
}

epidermidis occupied an intermediate position; and Proteus mirabilis and Klebsiella were resistant in 41-76\% of cases. Similar incidences of resistance were observed to sulphamethoxazole-trimethoprim, sulphamethoxazole, ampicillin, and nitrofurantoin.

These findings together with the rare occurrence of side effects and convenient dosage confirm the usefulness of plain trimethoprim for urinary tract infections.

\section{Introduction}

Combined treatment with sulphonamide and trimethoprim has been widely used for bacterial infections since 1969.1 Plain trimethoprim, however, has certain advantages over the combined regimen, including more convenient dosage and a low incidence of side effects, particularly with doses used for longterm prophylaxis against urinary tract infections. ${ }^{2}$ Nevertheless, an objection to using trimethoprim alone is the risk of increasing resistant bacteria, which might jeopardise the usefulness of the combined agent. ${ }^{2-4}$

Plain trimethoprim for long-term prophylaxis against urinary 